\title{
OPTIMAL PORTFOLIO SELECTION WITH TRANSACTION COSTS WHEN AN ILLIQUID ASSET PAYS CASH DIVIDENDS
}

\author{
BONG-GYU JANG
}

\begin{abstract}
We investigate an optimal portfolio selection problem with transaction costs when an illiquid asset pays cash dividends and there are constraints on the illiquid asset holding. We provide closed form solutions for the problem, and by using these solutions we illustrate interesting features of optimal policies.
\end{abstract}

\section{Introduction}

Consumption and portfolio optimization has been a central theme in financial research. This is because the optimization of consumption over lifetime under given constraints is the supreme objective of economic agents and the financial services industry exists to help them to find efficient way of financing optimized consumption. Due to development of continuous time finance theory it seems fair to say that we now understand the optimization problem in the absence of market frictions (e.g. taxes or transaction costs) pretty well (see [4], [12] and [13]). However, our knowledge about the consumption and investment problem in the presence of market frictions is still limited, because market frictions make it impossible or suboptimal for an investor to re-balance her portfolio continuously and this fact often prohibits a researcher from getting an explicit analytic solution for the investor's problem (see [5] and [6]).

In this note we investigate the optimal portfolio selection problem in the presence of transaction costs. Constantinides [3] started investigation of the problem in continuous time and showed that optimal strategy involves nontrading of an illiquid asset over a certain range of the ratio between the illiquid asset value and the liquid asset value. Davis and Norman [5] have succeeded in deriving a fully optimal strategy for the problem and providing a verification of optimality of their strategy. Liu and Loewenstein [11] have recently derived an optimal strategy analytically by considering an investor's problem in which the investor optimizes her utility of terminal wealth where her terminal time arrives

Received July 19, 2005.

2000 Mathematics Subject Classification. Primary 91B28, 90C39.

Key words and phrases. optimal portfolio, consumption, investment, dividend, transaction costs. 
randomly according to an exponential distribution. This note extends research by Constantinides and Liu and Loewenstein to include the case where an illiquid asset pays a stream of cash dividends and there are constraints on illiquid asset holdings. When there is a cost for trading an asset and consumption can only be purchased with the liquid asset, payment of cash dividends by the illiquid asset is expected to increase the attractiveness of the asset, and thereby increase the demand for the asset. We investigate this issue in this note.

We will treat four different models in this note:

Model 1. considers an investor who has a utility function for her bequest with a random investment horizon, which is exponentially distributed,

Model 2. considers an investor who has utility function for her consumption stream with an infinite investment horizon,

Model 3. is a general model containing both Model 1 and Model 2 as special cases, and

Model 4. is a model where the constraint 'investing on margin' described in [8] is added to Model 3.

We obtain closed-form solutions to the free boundary value problems arising from these models. Also by using these closed-form solutions, we illustrate interesting features of optimal policies.

\section{Models and results}

\subsection{General setting}

We consider a financial market where an investor can buy and sell two assets - one liquid asset (bond) and one illiquid asset (stock). The liquid asset is a riskfree asset with continuously compounded interest rate $r>0$ and the illiquid asset is a risky asset. The investor can buy the illiquid asset at the ask price $(1+\theta) S_{t}$, and sell it at the bid price $(1-\alpha) S_{t}$, where $0<\theta, \alpha<1$ are the transaction cost rates.

Throughout this note, assume all uncertainty is modelled by a probability space $(\Omega, \mathcal{F}, P)$ and a filtration $\left\{\mathcal{F}_{t}\right\}$ which satisfies the usual conditions. We assume that the price $S_{t}$ of the illiquid asset evolves according to the following stochastic differential equation:

$$
d S_{t}=\mu S_{t} d t+\sigma S_{t} d B_{t}
$$

where $\mu$ and $\sigma$ are positive constants and $\mu>r . B_{t}$ is a standard onedimensional Brownian motion which is adapted to the filtration $\left\{\mathcal{F}_{t}\right\}$.

Assume that the illiquid asset pays cash dividends continuously with a rate equal to $\delta$. The investor invests an amount $x_{t}$ in the liquid asset and an amount $y_{t}$ in the illiquid asset at time $t$. In order to simplify our analysis we assume the following borrowing constraint on the liquid asset

$$
x_{t} \geq 0, \forall t \geq 0
$$


although there is no difficulty for our analysis if it is replaced by the solvency condition

$$
x_{t}+(1-\alpha) y_{t} \geq 0, \forall t \geq 0 .
$$

Note that the fact $\mu>r$ guarantees $y_{t} \geq 0$ in optimum, as seen in [11].

\subsection{Model 1: utility for bequest}

Assume that the investor has an uncertain lifetime $\tau$ which is exponentially distributed with parameter $\lambda$, i.e.,

$$
P\{\tau \in d t\}=\lambda \exp (-\lambda t) d t .
$$

Therefore, the investor's average lifetime is $1 / \lambda$ and the variance of her lifetime is $1 / \lambda^{2}$ as seen in [2]. Also assume that this uncertain lifetime is independent of the illiquid stock process. Then the equations describing the evolutions of $x_{t}$ and $y_{t}$ are

$$
\begin{gathered}
d x_{t}=r x_{t} d t+\delta y_{t} d t-(1+\theta) d L_{t}+(1-\alpha) d U_{t}, x_{0}=x \\
d y_{t}=\mu y_{t} d t+\sigma y_{t} d B_{t}+d L_{t}-d U_{t}, y_{0}=y
\end{gathered}
$$

where $L_{t}$ and $U_{t}$ represent the cumulative purchase and sale of stock on time interval $[0, t]$, respectively. These are nondecreasing, right continuous, adapted to $\left\{\mathcal{F}_{t}\right\}$, and satisfying $L(0)=U(0)=0$. Let $\mathcal{A}(x, y)$ be the set of admissible policies $(L, U)$ that satisfy equations $(1)$ and $(2)$.

Under this set-up, the investor's problem is to maximize

$$
E\left[\left(x_{\tau}+(1-\alpha) y_{\tau}\right)^{1-\gamma} /(1-\gamma)\right]
$$

by choosing an admissible policy $(L, U)$. Here, $\gamma$ is the coefficient of the investor's relative risk aversion satisfying $\gamma>0, \gamma \neq 1$. The investor's value function is defined as

$$
v(x, y)=\sup _{(L, U) \in \mathcal{A}(x, y)} E\left[\frac{\left(x_{\tau}+(1-\alpha) y_{\tau}\right)^{1-\gamma}}{1-\gamma}\right] .
$$

By using the distribution of uncertain lifetime $\tau$, we can rewrite this equation as

$$
v(x, y)=\sup _{(L, U) \in \mathcal{A}(x, y)} E\left[\int_{0}^{\infty} e^{-\lambda t} \frac{\lambda\left(x_{t}+(1-\alpha) y_{t}\right)^{1-\gamma}}{1-\gamma} d t\right] .
$$

Finally we assume that the investor's initial holdings in bond and stock are constrained to lie in the first quadrant

$$
F=\left\{(x, y) \in \mathbb{R}^{2}: x>0, y>0\right\} .
$$

Then it is well-known that $v$ is concave, strictly increasing and homothetic in $F$, that is, homogeneous of degree $1-\gamma$. (See Theorem 1 in [10], pp.155.)

As seen in [5], $F$ splits into three regions, buy region $(B)$, sell region $(S)$ and no-transaction region $(N T)$, and the optimal transaction strategies are bangbang, namely, buying and selling the stock either takes place at a maximum 


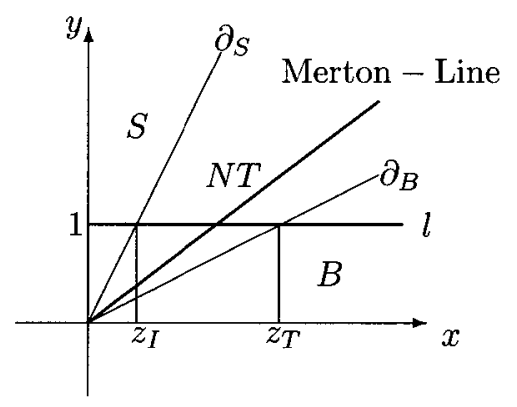

Figure 1. the regions $S, B, N T$, and $l, z_{I}, z_{T}$

rate in $S$ and $B$ or not at all in $N T$. The boundaries of these regions are straight lines through the origin. Set $\partial_{S}\left(\partial_{B}\right)$ to be such straight line between $S(B$, resp.) and $N T$.

In $N T$, we obtain the Hamilton-Jacobi-Bellman (HJB) equation of the value function $v$ in (3). The HJB equation takes the following form

$$
\frac{1}{2} \sigma^{2} y^{2} v_{y y}+(r x+\delta y) v_{x}+\mu y v_{y}-\lambda v+\frac{\lambda(x+(1-\alpha) y)^{1-\gamma}}{1-\gamma}=0 .
$$

In $S$ we obtain

$$
(1-\alpha) v_{x}=v_{y}
$$

and in $B$,

$$
(1+\theta) v_{x}=v_{y}
$$

Here, $v_{x}=\frac{\partial v}{\partial x}, v_{y}=\frac{\partial v}{\partial y}$ and $v_{y y}=\frac{\partial^{2} v}{\partial y^{2}}$.

The homogeneity of $v$ implies that $v$ can be expressed by using a one-variable function $\psi$ as follows:

$$
v(x, y)=y^{1-\gamma} \psi\left(\frac{x}{y}\right)
$$

so we can treat only the straight line

$$
l \equiv\left\{(x, y) \in \mathbb{R}^{2}: y=1, x>0\right\}
$$

instead of the whole first quadrant $F$. By $\partial_{S}$ and $\partial_{B}$, the line $l$ is divided into three open intervals, whose $x$-coordinates are defined by $\left(0, z_{I}\right),\left(z_{I}, z_{T}\right)$ and $\left(z_{T}, \infty\right)$, respectively. (See Figure 1.)

Therefore, if we let $z_{t}=x_{t} / y_{t}$ and $z_{0}=z$, we can rewrite equations (4), (5) and (6) as the following simple ordinary differential equation (ODE):

$$
\beta_{2} z^{2} \psi^{\prime \prime}(z)+\beta_{1} z \psi^{\prime}(z)+\delta \psi^{\prime}(z)+\beta_{0} \psi(z)+\lambda \frac{(z+1-\alpha)^{1-\gamma}}{1-\gamma}=0
$$


where $\beta_{2}=\frac{1}{2} \sigma^{2}, \beta_{1}=\gamma \sigma^{2}-(\mu-r), \beta_{0}=\frac{1}{2} \sigma^{2} \gamma(\gamma-1)+\mu(1-\gamma)-\lambda$, if $z \in\left(z_{I}, z_{T}\right)$,

$$
\begin{aligned}
& (z+1-\alpha) \psi^{\prime}(z)=(1-\gamma) \psi(z), \text { if } z \in\left(0, z_{I}\right), \\
& (z+1+\theta) \psi^{\prime}(z)=(1-\gamma) \psi(z), \text { if } z \in\left(z_{T}, \infty\right)
\end{aligned}
$$

Finally, note that the investor's optimal portfolio choice problem has become the free boundary value problem with free boundaries $z_{I}$ and $z_{T}$. By the smooth pasting conditions, we know that the solution of the problem must be in the class of $C^{2}$-functions. Equation (8) becomes

$$
\psi^{\prime \prime}(z)+a \frac{1}{z} \psi^{\prime}(z)+b \frac{1}{z^{2}} \psi^{\prime}(z)-c \frac{1}{z^{2}} \psi(z)+\frac{\lambda(z+1-\alpha)^{1-\gamma}}{\beta_{2} z^{2}(1-\gamma)}=0,
$$

where $a=\beta_{1} / \beta_{2}, b=\delta / \beta_{2}$ and $c=-\beta_{0} / \beta_{2}$.

Theorem 2.1. Suppose $(a-1)^{2}+4 c \geq 0$ is not a square. Then the general solution of the second order $O D E(8)$ is

$$
C_{1} \psi_{1}(z)+C_{2} \psi_{2}(z)+\psi_{p}(z) \text {, for some constants } C_{1} \text { and } C_{2},
$$

where

$$
\begin{aligned}
& \psi_{1}(z)=M\left(N^{-}, 2 N^{-}+2-a, b / z\right) \cdot\left(\frac{b}{z}\right)^{N^{-}} \\
& \psi_{2}(z)=M\left(N^{-}, 2 N^{+}+2-a, b / z\right) \cdot\left(\frac{b}{z}\right)^{N^{+}}
\end{aligned}
$$

for $N^{ \pm}=\frac{1}{2}\left(a-1 \pm \sqrt{(a-1)^{2}+4 c}\right)$, and

$$
\psi_{p}(z)=\int_{z_{I}}^{z} \frac{\psi_{1}(t) \psi_{2}(z)-\psi_{1}(z) \psi_{2}(t)}{\psi_{1}^{\prime}(t) \psi_{2}(t)-\psi_{1}(t) \psi_{2}^{\prime}(t)} \frac{\lambda(t+1-\alpha)^{1-\gamma}}{\beta_{2}(1-\gamma) t^{2}} d t
$$

Here, the function $M(\cdot, \cdot, \cdot)$ is the confluent hypergeometric function, i.e., the Kummer function (see in [14]).

Proof. We guess a homogeneous solution of (11)

$$
\psi(z)=\left(\frac{b}{z}\right)^{N} \cdot f\left(\frac{b}{z}\right)
$$

with some function $f$. Then, after some calculations, we obtain

$$
\begin{aligned}
\psi^{\prime \prime}(z)+a \frac{1}{z} \psi^{\prime}(z)+ & b \frac{1}{z^{2}} \psi^{\prime}(z)-c \frac{1}{z^{2}} \psi(z) \\
= & -\frac{1}{z^{4}}\left(\frac{b}{z}\right)^{N}\left[(b N+(c+(a-1-N) N) z) z f\left(\frac{b}{z}\right)\right. \\
& \left.+b\left((b+(a-2-2 N) z) f^{\prime}\left(\frac{b}{z}\right)-b f^{\prime \prime}\left(\frac{b}{z}\right)\right)\right] .
\end{aligned}
$$

If $c+(a-1-N) N=0$, i.e., $N=\frac{1}{2}\left(a-1 \pm \sqrt{(a-1)^{2}+4 c}\right)$,

$$
(\mathrm{RHS})=-\frac{b}{z^{3}}\left(\frac{b}{z}\right)^{N}\left[N f\left(\frac{b}{z}\right)+\frac{b}{z} f^{\prime}\left(\frac{b}{z}\right)-(2 N+2-a) f^{\prime}\left(\frac{b}{z}\right)-\frac{b}{z} f^{\prime \prime}\left(\frac{b}{z}\right)\right] .
$$


Also if we let $f(z)=M(N, 2 N+2-a, z)$, we know that it satisfies the Kummer differential equation

$$
N f\left(\frac{b}{z}\right)+\frac{b}{z} f^{\prime}\left(\frac{b}{z}\right)-(2 N+2-a) f^{\prime}\left(\frac{b}{z}\right)-\frac{b}{z} f^{\prime \prime}\left(\frac{b}{z}\right)=0 .
$$

Thus we have found two homogeneous solutions. A general solution is obtained immediately by using the method of variation of parameters. We refer to [1] for this method.

In Theorem 2.1, the assumptions for $(a-1)^{2}+4 c$ are necessary in order to get real $N^{ \pm}$and guarantee the existence of the function $M$. The following corollary can immediately be derived from the theorem.

Corollary 2.2. Suppose all assumptions in Theorem 2.1 are valid. If $0<$ $z_{I}<z_{T}<\infty$, then $\psi$ is of the following form,

$$
\psi(z)= \begin{cases}A \frac{(z+1-\alpha)^{1-\gamma}}{1-\gamma} & \text { if } 0<z \leq z_{I} \\ C_{1} \psi_{1}(z)+C_{2} \psi_{2}(z)+\psi_{p}(z) & \text { if } z_{I}<z<z_{T} \\ B \frac{(z+1+\theta)^{1-\gamma}}{1-\gamma} & \text { if } z \geq z_{T}\end{cases}
$$

for six constants $A, B, C_{1}, C_{2}, z_{I}$ and $z_{T}$.

Remark 2.3. The six unknowns of constants $A, B, C_{1}, C_{2}$ and free boundaries $z_{I}, z_{T}$ can be determined by $C^{2}$-conditions at the free boundaries (thus they are six). We call these the smooth pasting conditions or super contact conditions, which are minutely described in [7]. Using these conditions, we can derive six algebraic equations with respect to the six unknowns and these can be solved numerically. The argument similar to this can be shown in [11]. Furthermore, the argument can be used to determine the unknowns described in Corollary 2.4 (Section 2.3) and Corollary 2.6 (Section 2.4).

If $z_{T}$ is infinite, we need two more limiting conditions as seen in Theorem 1 in [11]. The reader can easily find such conditions. Also in [11], Liu and Loewenstein have mentioned that they obtained a closed-form solution of the problem. But they have not shown it explicitly in their article, as we do here.

\subsection{Model 2: utility for consumption}

Now we explore a model, where the investor has an infinite investment horizon and consume continuously with rate $c_{t}$ at time $t$. Then the equation describing the evolution of $x_{t}$ is

$$
d x_{t}=r x_{t} d t-c_{t} d t+\delta y_{t} d t-(1+\theta) d L_{t}+(1-\alpha) d U_{t}, x_{0}=x,
$$

and that of $y_{t}$ is as in (2). Assume that the set $\mathcal{A}(x, y)$ of admissible policies $(L, U)$ satisfies equations (2) and (14), and the initial endowment $(x, y)$ is in $F$. 
In this model, the investor's problem is to maximize

$$
E\left[\int_{0}^{\infty} e^{-\rho t} \frac{c_{t}^{1-\gamma}}{1-\gamma} d t\right]
$$

where $\rho>0$ is her subjective discount rate. The investor's value function is defined in the same way as in the previous section, i.e., it is the supremum of the investor's utility obtainable with an admissible policy and can be considered as maximum happiness obtained from discounted consumption stream over the investment horizon.

In his seminal paper [3] Constantinides considered only the following consumption policies: an investor's consumption rate is a constant fraction of her liquid asset holding. Following him, we assume

$$
c_{t}=\kappa x_{t} \text { for } 0<\kappa<1 .
$$

Davis and Norman [5] has shown that the consumption policy is a suboptimal policy if we think of $c_{t}$ as a control variable. However, we make the assumption to derive a closed-form solution.

The optimal policy is also a bang-bang policy and the HJB equation in the $N T$ region becomes

$$
\frac{1}{2} \sigma^{2} y^{2} v_{y y}+[(r-\kappa) x+\delta y] v_{x}+\mu y v_{y}-\rho v+\frac{(\kappa x)^{1-\gamma}}{1-\gamma}=0
$$

and the change of variable formula (7) yields

$$
\beta_{2} z^{2} \psi^{\prime \prime}(z)+\beta_{1} z \psi^{\prime}(z)+\delta \psi^{\prime}(z)+\beta_{0} \psi(z)+\frac{(\kappa z)^{1-\gamma}}{1-\gamma}=0,
$$

where (redefined) parameters $\beta_{2}=\frac{1}{2} \sigma^{2}, \beta_{1}=\gamma \sigma^{2}-(\mu-r+\kappa), \beta_{0}=\frac{1}{2} \sigma^{2} \gamma(\gamma-$ $1)+\mu(1-\gamma)-\rho$, if $z \in\left(z_{I}, z_{T}\right)$. Also $\psi(z)$ satisfies $(9)$ on $\left(0, z_{I}\right)$ and $(10)$ on $\left(z_{T}, \infty\right)$. As a result we derive the following corollary from Theorem 2.1.

Corollary 2.4. Suppose $a=\beta_{1} / \beta_{2}, b=\delta / \beta_{2}$ and $c=-\beta_{0} / \beta_{2}$ and all assumptions in Theorem 2.1 are valid. If $0<z_{I}<z_{T}<\infty, \psi$ is of the same form as in (13), where $\psi_{1}$ and $\psi_{2}$ are also as in (12) and

$$
\psi_{p}(z)=\int_{z_{I}}^{z} \frac{\psi_{1}(t) \psi_{2}(z)-\psi_{1}(z) \psi_{2}(t)}{\psi_{1}^{\prime}(t) \psi_{2}(t)-\psi_{1}(t) \psi_{2}^{\prime}(t)} \frac{(\kappa t)^{1-\gamma}}{\beta_{2}(1-\gamma) t^{2}} d t
$$

Remark 2.5. If we think of $c_{t}$ in the class of consumption rates of the form (15) as a control variable in this problem, we may obtain the value function by finding $\kappa$ which maximizes $\psi(z)$ for all $z \in[0, \infty)$. But proving the existence of such a global constant $\kappa$ is still an open problem. Constantinides [3] has calculated $\kappa$ which maximizes $\psi\left(z_{M}\right)$ where $z_{M}$ is the optimal portfolio proportion $x / y$ in the absence of transaction costs. 


\subsection{Model 3: a general model}

Assume that the set $\mathcal{A}(x, y)$ of admissible policies $(L, U)$ that satisfy equations (2) and (14), $(x, y) \in F$, and the consumption rate $c_{t}$ follows (15). The value function of this model is

$$
\begin{aligned}
v(x, y)= & \sup _{(L, U) \in \mathcal{A}(x, y)} E\left[\int _ { 0 } ^ { \infty } e ^ { - ( \rho + \lambda ) t } \left((1-\varepsilon) \frac{c_{t}^{1-\gamma}}{1-\gamma}\right.\right. \\
& \left.\left.+\varepsilon \lambda \frac{\left(x_{t}+(1-\alpha) y_{t}\right)^{1-\gamma}}{1-\gamma}\right) d t\right] \quad \text { for } 0 \leq \varepsilon \leq 1,
\end{aligned}
$$

where $\rho$ is a subjective time discount rate and $\lambda$ a mortality rate. Note that utility of bequest with weight $\varepsilon$ and utility of consumption with weight $(1-\varepsilon)$ compose the investor's utility function. The reader also can find this type of utility function in [9]. Following the argument in subsection 2.2 or 2.3 , we can obtain the corollary below. Here, $\beta_{2}=\frac{1}{2} \sigma^{2}, \beta_{1}=\gamma \sigma^{2}-(\mu-r+\kappa), \beta_{0}=$ $\frac{1}{2} \sigma^{2} \gamma(\gamma-1)+\mu(1-\gamma)-\rho-\lambda$.

Corollary 2.6. Suppose $a=\beta_{1} / \beta_{2}, b=\delta / \beta_{2}$ and $c=-\beta_{0} / \beta_{2}$ and all assumptions in Theorem 2.1 are valid. If $0<z_{I}<z_{T}<\infty, \psi$ is of the same form as in (13), where $\psi_{1}$ and $\psi_{2}$ are also in (12) and

$$
\psi_{p}(z)=\int_{z_{I}}^{z} \frac{\psi_{1}(t) \psi_{2}(z)-\psi_{1}(z) \psi_{2}(t)}{\psi_{1}^{\prime}(t) \psi_{2}(t)-\psi_{1}(t) \psi_{2}^{\prime}(t)} \cdot \frac{(1-\varepsilon)(\kappa t)^{1-\gamma}+\varepsilon \lambda(t+1-\alpha)^{1-\gamma}}{\beta_{2}(1-\gamma) t^{2}} d t
$$

\subsection{Model 4: with a portfolio constraint}

In Model 4 we add a portfolio constraint to Model 3 . The constraint is given by the requirement that a constant fraction $0<\eta<1$ of the investor's total wealth is the maximum amount that can be invested in the illiquid asset, more specifically,

$$
y_{t} \leq \eta\left(x_{t}+(1-\alpha) y_{t}\right) \text { or equivalently } z_{t} \geq \frac{1}{\eta}+\alpha-1 \text { for all } t>0 .
$$

This is a special case of the restriction given in [8]. Note that the investor's initial endowment $(x, y) \in F$ may not satisfy the constraint, that is, $(x, y) \in$ $\{(a, b) \in F: b>\eta(a+(1-\alpha) b)\}$. If so, the initial transaction should contain the policy of selling the illiquid asset at a maximum speed so that the investor's portfolio satisfies (16), as well as a bang-bang policy.

Let $\hat{\eta}$ be $(1 / \eta+\alpha-1)$ in (16). If $z_{I}^{*}$ is a sell boundary in Corollary 2.6 and $\hat{\eta} \leq z_{I}^{*}$, then the optimal policy does not change at all, since the sell region in the absence of the portfolio constraint contains the whole restricted area. Otherwise, the sell boundary should be $\hat{\eta}$. According to the argument in [7], the value function satisfies only the first-derivative continuity, derived from value matching condition, at $z=\hat{\eta}$. 


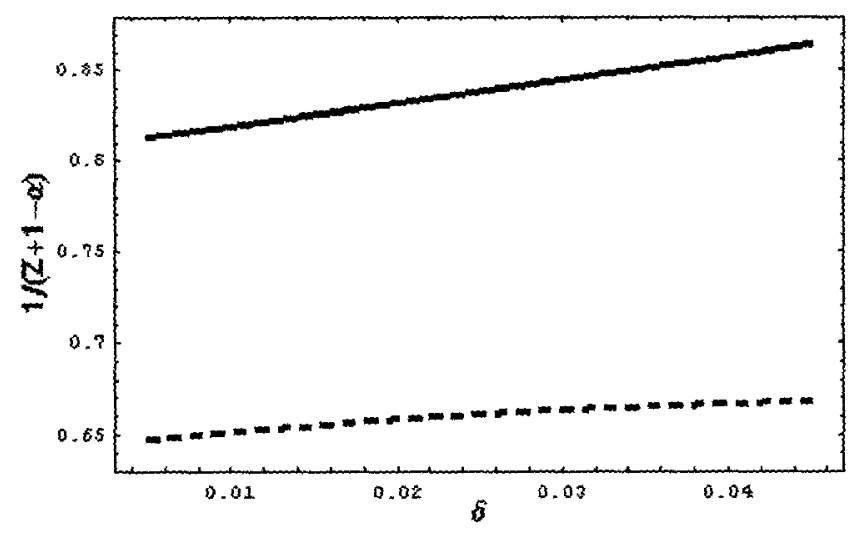

FIGURE 2. The ratio of the investor's holdings of the illiquid asset at the sell boundary (the solid line) and at buy boundary (the dotted line) to her liquidated wealth as a function of the dividend rate in Model 1, for parameters $\mu+\delta=0.16, \sigma=0.2$, $\lambda=0.1, \gamma=2, r=0.1$, and $\theta=\alpha=0.01$.

Corollary 2.7. Suppose all assumptions in Corollary 2.6 are valid.

(a) Assume that $z_{I}^{*}$ is the sell boundary in Corollary 2.6. If $\hat{\eta} \leq z_{1}^{*}, \psi$ is exactly the same as in Corollary 2.6 .

(b) Otherwise, $\psi$ is the same form as in Corollary 2.6 with $z_{Y}$ being replaced by $\hat{\eta}$.

Remark 2.8. Different from the previous models, we need to determine only five unknowns in this case. Three smooth pasting conditions ( $C^{2}$-condition) at $z=z_{T}$ and two value matching conditions $\left(C^{1}\right.$-condition) at $z=\hat{\eta}$ enable us to calculate them numerically,

Remark 2.9. We have omitted the verification theorem of each model, since this note is only focused on finding closed-form solution of the free boundary value problem arising from each. We confirm that it is established by using the arguments in $\{5\},\{9\}$ and $[11\}$.

\section{Numerical results}

Figure 2 presents the ratio of the investor's holdings of the illiquid asset on the sell boundary (the solid line) and on buy boundary (the dotted line) to her liquidated wealth as a function of the dividend yield in Model 1, for parameters $\mu+\delta=0.16, \sigma=0.2, \lambda=0.1, \gamma=2, r=0.1$, and $\theta=\alpha=0.01$. Since $\mu+\delta$ is fixed, if there is no transaction costs, we think of the illiquid asset as a risky asset having a constant expected rate of return $\mu+\delta$ and no dividend. In this case, the Merton line, the amount of investor's optimal holdings of the illiquid asset to her total wealth, does not change at all as $\delta$ changes. However in the 


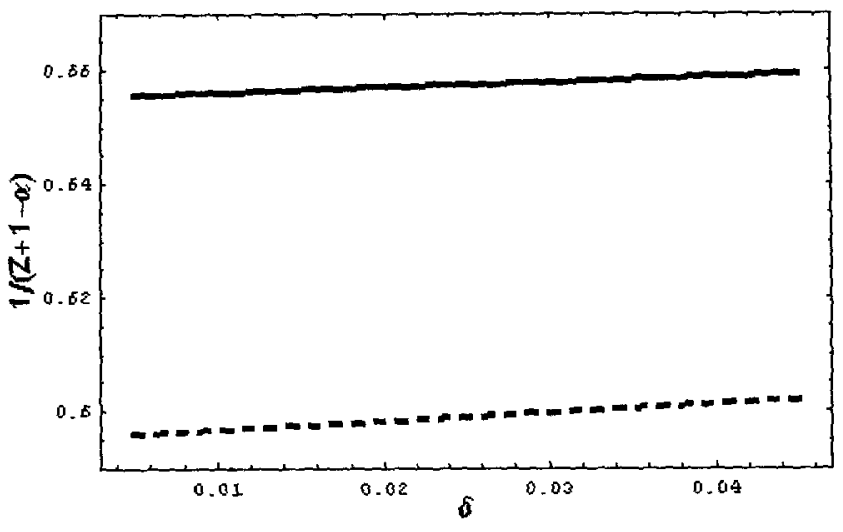

FIguRE 3. The ratio of the investor's holdings of the illiquid asset at the sell boundary (the solid line) and at buy boundary (the dotted line) to her liquidated wealth as a function of the dividend rate in Model 2, for parameters $\mu+\delta=0.16, \sigma=0.2$, $\rho=0.1, \gamma=2, r=0.1, \kappa=0.3$ and $\theta=\alpha=0.01$.

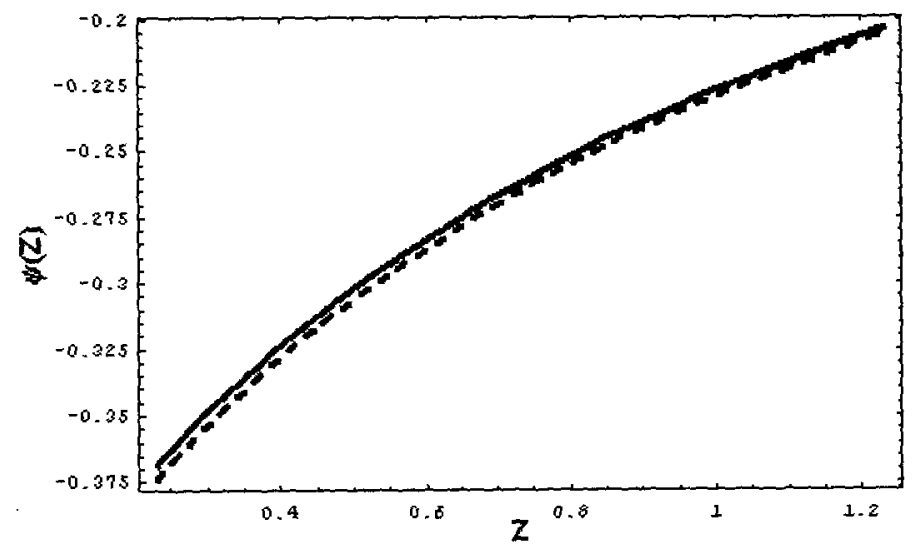

FIGURE 4. The function $\psi(z)$ with no leverage constraint (the solid line) and with leverage constraint $\hat{\eta}=1$ (the dotted line) in Model 4, for parameters $\mu=0.15, \delta=0.01, \sigma=0.2$, $\lambda=0.1, \rho=0, \gamma=2, r=0.1, \varepsilon=1$ and $\theta=\alpha=0.01$.

Figure, the amounts of investor's holdings of the illiquid asset to her liquidated wealth on both the sell and buy boundaries increase as $\delta$ increases. This means that, as the dividend yield increases, the investor invests more in the illiquid asset. Also, we can see that Figure 3 for Model 2 gives the result same as this. 




FiguRE 5 . The length of the interval $\left[1 /\left(z_{T}+1-\alpha\right), 1 /\left(z_{I}+\right.\right.$ $1-\alpha)]$ as a function of $\hat{\eta}$ in Model 4 , for parameters $\mu=$ $0.15, \delta=0.01, \sigma=0.2, \lambda=0.1, \rho=0, \gamma=2, r=0.1, \varepsilon=1$ and $\theta=\alpha=0.01$.

Figure 4 presents the function $\psi(z)$ with no portfolio constraint (the solid line) and with constraint parameter $\hat{\eta}=1$ (the dotted line), in Model 4, for parameters $\mu=0.15, \delta=0.01, \sigma=0.2, \lambda=0.1, \rho=0, \gamma=2, r=0.1, \varepsilon=1$ and $\theta=\alpha=0.01$. We can immediately observe that the portfolio constraint yields the reduction of the investor's maximum expected utility.

Figure 5 presents the length of the interval $\left[1 /\left(z_{T}+1-\alpha\right), 1 /\left(z_{I}+1-\alpha\right)\right]$ corresponding to the $N T$ region as a function of $\hat{\eta}$ in Model 4, for parameters $\mu=0.15, \delta=0.01, \sigma=0.2, \lambda=0.1, \rho=0, \gamma=2, r=0.1, \varepsilon=1$ and $\theta=\alpha=0.01$. We observe that, if $\hat{\eta}<z_{I}^{*}$ (i.e., $z_{I}^{*}=z_{I}$ ) the length does not change, but otherwise(i.e., $\hat{\eta}=z_{I}$ ) it decreases as $\hat{\eta}$ increases.

Acknowledgement. I thank Hyeng Keun Koo for helpful comments.

\section{References}

[1] W. E. Boyce and R. C. DiPrima, Elementary Differential Equations and Boundary Value Problems, John Wiley \& Sons, New York, 1965.

[2] P. Carr, Randomization and the American Put, Review of Financial Studies 11 (1998), $597-626$.

[3] G. M. Costantinides, Capital Market Equilibrium with Transaction Costs, Journal of Political Economy 94 (1986), 842-862.

[4] D. Cuoco, Optimal Consumption and Equilibrium Prices with Portfolio Constraints and Stochastic Income, J. Econom. Theory 72 (1997), no. 1, 33-73.

[5] M. H. A. Davis and A. R. Norman, Portfolio Selection with Transaction Costs, Math. Oper. Res. 15 (1990), no. 4, 676-713.

[6] V. DeMiguel and R. Uppal, Portfolio Investment with the Exact Tax Basis via Nonlinear Programming, Management Science, Forthcoming. 
[7] B. Dumas, Super Contact and Related Optimality Conditions, J. Econom. Dynam. Control 15 (1991), no. 4, 675-685.

[8] S. J. Grossman and J.-L. Vila, Optimal Dynamic Trading with Leverage Constraints, Journal of Financial and Quantitative Analysis 27 (1992), 151-163.

[9] B.-G. Jang, H. K. Koo, H. Liu and M. Loewenstein, Liquidity Premia and Transaction Costs, 2006 AFA (American Finance Association) Boston meetings, 2005, http://www.olin.wustl.edu/faculty/liuh/Papers/Regime_October_05.pdf.

[10] R. Korn, Optimal Portfolios, World Scientific, 1997, 151-171.

[11] H. Liu and M. Loewenstein, Optimal Portfolio Selection with Transaction Costs and Finite Horizons, Review of Financial Studies 15 (2002), 805-835.

[12] R. C. Merton, Optimum Consumption and Portfolio Rules in a Continuous-Time Model, J. Econom. Theory 3 (1971), no. 4, 373-413.

[13] M. Schroder and C. Skiadas, Optimal Consumption and Portflio Selection with Stochastic Differential Utility, J. Econom. Theory 89 (1999), no. 1, 68-126.

[14] N. M. Temme, Special Functions. An introduction to the classical functions of mathematical physics, John Wiley \& Sons, New York, 1996.

Derivatives SuPERVISION TEAM

Financial Supervisory Service

SeOul 150-743, KoreA

E-mail address: bonggyujang@kaist.ac.kr 